\title{
Características Sociodemográficas y Depresión en Adolescentes de Escuelas Secundarias Públicas del Municipio Camerino Z. Mendoza, Veracruz
}

\author{
Abril Camarillo-Quiroz, L.E. \\ Estudiante de Maestría en Ciencias de Enfermería, \\ Universidad Autónoma de Querétaro \\ María del Carmen García-de-Jesús, M.E. \\ Profesor de la Facultad de Enfermería de la \\ Universidad Autónoma de Querétaro \\ Uriel Alejandro Morales Carrera, M.A.N. \\ Docente del Instituto Tecnológico Superior de Zongolica
}

URL:http://dx.doi.org/10.19044/esj.2020.v16n2p1

\section{Resumen}

La depresión en los adolescentes es un problema de salud pública que afecta a miles de jóvenes en el mundo. Esta investigación tiene como objetivo identificar las características sociodemográficas de los adolescentes en las escuelas públicas y su nivel de depresión. El enfoque de investigación es mixto, el instrumento aplicado se llama Inventario de depresión infantil (CDI) creado por Kovacs (2004) y se aplicó a estudiantes de secundaria del municipio de Camerino Z. Mendoza, Veracruz, México. El instrumento fue validado con Alfa de Cronbach y el análisis estadístico de las variables y tablas se realizó con el software SPSS versión 20. Como resultado, se identificaron las características sociodemográficas de los adolescentes con síntomas de depresión. Se concluye que la adolescencia es una edad vulnerable en la que puede desarrollarse la depresión, por lo que es importante que las instituciones sigan los programas de identificación de factores de riesgo y brinden apoyo profesional oportuno.

Palabras claves: Depresión, adolescencia, educación 


\title{
Sociodemographic Characteristics of Depression in Adolescents of Public Secondary Schools in the Municipality of Camerino Z. Mendoza, Veracruz
}

\author{
Abril Camarillo-Quiroz, L.E. \\ Estudiante de Maestría en Ciencias de Enfermería, \\ Universidad Autónoma de Querétaro \\ María del Carmen García-de-Jesús, M.E. \\ Profesor de la Facultad de Enfermería de la \\ Universidad Autónoma de Querétaro \\ Uriel Alejandro Morales Carrera, M.A.N. \\ Docente del Instituto Tecnológico Superior de Zongolica
}

\begin{abstract}
Depression in adolescents is a public health problem that affects thousands of young people around the world. This paper focuses on identifying the sociodemographic characteristics of adolescents in public schools and their level of depression. The research approach is mixed, and the instrument applied is called Child Depression Inventory (CDI) created by Kovacs (2004). This instrument was applied to high school students of the municipality of Camerino Z. Mendoza, Veracruz, Mexico. The instrument was validated with Cronbach's Alpha and the statistical analysis of the variables and tables was performed using SPSS version 20 software. As a result, the sociodemographic characteristics of adolescents with symptoms of depression were identified. It was concluded that since the adolescent age is a vulnerable age at which depression can develop, it is important that institutions follow risk factor identification programs and provide timely professional support.
\end{abstract}

Keywords: Depression, adolescence, education

\section{Introduction}

La depresión es un problema mundial que tiene impacto en todas y cada una de las esferas de la sociedad, se conceptualiza como un trastorno emocional que se caracteriza por alteraciones de humor, tristeza, baja autoestima, inhibición, fatiga, insomnio, pensamientos negativos, y tiene como consecuencia la disminución de serotonina, lo cual impide el desarrollo de actividades de la vida diaria con normalidad (Tijerina \& Zandra 2019). 
La Organización Mundial de la Salud (OMS) estima que la depresión afecta a más de 350 millones de personas de todas las edades y en todas las comunidades, afectando dos veces más a mujeres con respecto a los hombres, contribuyendo a la carga mundial de morbilidad y mortalidad (Morocho, 2014). La depresión es un trastorno emocional que puede presentarse en etapas tempranas de la vida, continuando en la adolescencia y puede extenderse a la edad adulta (Berenzon, Lara, Robles \& Medina-Mora, 2013).

Berenzon et al. (2013) indica que en el mundo la depresión representa la cuarta causa de discapacidad en cuanto a la pérdida de años de vida saludables y en México ocupa el primer lugar de discapacidad para las mujeres $\mathrm{y}$ el noveno para los hombres. Tiene una alta co-ocurrencia con otros trastornos como la ansiedad, el consumo de sustancias, la diabetes y las enfermedades cardiacas; a pesar del impacto que tiene este trastorno, una gran proporción de personas no acude a tratamiento, retrasa mucho la búsqueda de ayuda, o bien no recibe la asistencia adecuada (Ortiz, 2012).

Existen varios tipos de trastornos depresivos, los más comunes son el trastorno depresivo grave y el trastorno distímico.

El trastorno depresivo grave, también llamado depresión grave, se caracteriza por una combinación de síntomas que interfieren con la capacidad para trabajar, dormir, estudiar, comer, y disfrutar de las actividades que antes resultaban placenteras; incapacita a la persona y le impide desenvolverse con normalidad. Este trastorno se divide en trastorno depresivo mayor de episodio único (sólo un episodio depresivo mayor) y trastorno depresivo mayor de episodio recurrente (con un intervalo de dos meses consecutivos entre episodios que no cumplen el criterio de episodio depresivo mayor).

Se caracteriza por presentar un estado de ánimo depresivo la mayor parte del día, por una disminución de interés hacia actividades que suponían placer a la persona, pérdida o aumento de peso importante, alteraciones del sueño (insomnio o hipersomnia), agitación o enlentecimiento motor, fatiga o pérdida de energía, sentimientos de inutilidad o de culpa excesivos, dificultades para concentrarse, pensamientos recurrentes de muerte o ideación suicida (Ferrer, 2015).

El trastorno distímico, también llamado distimia, se caracteriza porque sus síntomas son de larga duración (dos años o más), aunque menos graves, pueden no incapacitar a una persona, pero sin impedirle desarrollar una vida normal o sentirse bien. Las personas con distimia también pueden padecer uno o más episodios de depresión grave a lo largo de sus vidas, algunas formas de trastorno depresivo muestran características levemente diferentes a las descritas anteriormente o pueden desarrollarse bajo circunstancias únicas (Berenzon et al.). Se caracteriza por baja autoestima, sentimientos de desesperanza en una duración mínima de dos años frente a las dos semanas que requiere el episodio depresivo mayor (Ferrer, 2015). 
De acuerdo a Berenzon et al. (2013) existen otros tipos de depresión como son:

Depresión psicótica, que ocurre cuando una enfermedad depresiva grave está acompañada por alguna forma de psicosis, tal como ruptura con la realidad, alucinaciones, y delirios.

Depresión posparto, la cual se diagnóstica si una mujer que ha dado a luz recientemente sufre un episodio de depresión grave dentro del primer mes después del parto. Se calcula que del 10 al 15 por ciento de las mujeres padecen depresión posparto luego de dar a luz.

Trastorno afectivo estacional, se caracteriza por la aparición de una enfermedad depresiva durante los meses del invierno, cuando disminuyen las horas de luz solar. La depresión generalmente desaparece durante la primavera y el verano.

La depresión está presente en diferentes etapas del ciclo vital, uno de los periodos de mayor riesgo para su aparición es la adolescencia, dada la vulnerabilidad de esta etapa, además del riesgo de cronicidad de dicha enfermedad cuando se inicia a edades tempranas, pues más del 3\% de los adolescentes sufre condiciones depresivas tan graves que pueden ocasionar en ellos diversas disfunciones sociales (Jiménez, Wagner \& González-Forteza, 2015).

La adolescencia es una etapa de transición, y en esta etapa los cambios cuantitativamente, se dan en una proporción acelerada y cualitativamente se observa en el desarrollo de una nueva organización de la personalidad psicológica y social, acompañadas de necesidades, motivaciones, capacidades e intereses (Vivar \& Brito, 2015).

Según Rivera-Heredia, Martínez-Servín y Obregón-Velasco (2013) en el Plan Integral de soporte a la infancia y a la adolescencia de la Generalitat de Catalunya, la mayoría de autores, que han estudiado los diferentes cambios que se producen en los seres humanos a lo largo de su ciclo vital, consideran la adolescencia como un período de tránsito entre la infancia y la edad adulta, iniciando este periodo con la pubertad, momento en el que se producen una serie de cambios físicos y hormonales en el organismo que a su vez propician toda una serie de cambios psicológicos y sociales. Estos cambios sitúan a los chicos y chicas en un periodo de búsqueda de la propia identidad y de reconstrucción constante de la propia personalidad en el intento de dejar de ser niños o niñas para convertirse en adultos.

Amores (2012) indica que la OMSS define la adolescencia como el periodo de vida el cual el individuo adquiere madurez reproductiva, transita los patrones psicológicos de la niñez a la adultez y establece su independencia socioeconómica.

Los adolescentes constituyen uno de los grupos etarios que presenta mayor probabilidad de sufrir síntomas depresivos, ya que se ha mencionado 
en dicha etapa se llevan a cabo procesos de cambios físico, cognitivo, afectivo y sociocultural, que demandan de los jóvenes el desarrollo de estrategias de afrontamiento que les permitan establecer un sentido de identidad, autonomía, así como éxito personal y social. Además, es el periodo de desarrollo más importante para la adquisición y el mantenimiento de patrones de comportamiento saludable, que permiten disminuya el riesgo y prevengan el desarrollo de trastornos clínicos durante este periodo y la edad adulta (Romero, Guajardo, Ibarra \& Castro, 2013).

Ferrer (2015) indica que la prevalencia de la depresión en esta edad llega a alcanzar niveles similares a los obtenidos en la edad adulta, sugiriendo que la depresión en los adultos puede estar precedida por episodios depresivos en la adolescencia. De ahí la importancia de identificar los factores de vulnerabilidad a la depresión y de realizar una prevención e intervención para evitar la recurrencia de los episodios depresivos en la edad adulta.

\section{Método}

El tipo de investigación es observacional con un diseño de estudio de tipo descriptivo, transversal y correlacional en adolescentes de nivel de educación secundaria en escuelas públicas, se aplicó el instrumento CDI (Children's Depression Inventory) o Inventario de Depresión Infantil, de M. Kovacs (2014) siendo el test más utilizando en el mundo para identificar depresión infantil, con una confiabilidad que oscila entre 0.71 y 0.94 mediante Alfa de Cronbach.

La determinación de la muestra es probabilística por proporción, siendo su selección de forma aleatoria simple. Con una población de 1631 alumnos a nivel secundaria en el municipio de Camerino Z Mendoza según datos del Sistema interactivo de Consulta Estadística Educativa (2019).

Para obtener la muestra se aplicó la fórmula de muestreo de poblaciones finitas:

Dónde: • $\mathrm{N}=$ Total de la población (1631)

- $\mathrm{Z} \alpha=1.96$ al cuadrado (si la seguridad es del 95\%)

- $\mathrm{p}=$ proporción esperada (en este caso $50 \%=0.5$ )

- $\mathrm{q}=1-\mathrm{p}($ en este caso $1-0.5=0.5)$

- $\mathrm{d}=$ precisión (en su investigación use un 5.66\%).

$$
\boldsymbol{n}=\frac{\mathbf{z}^{2} \cdot \mathbf{N} \cdot \mathbf{p} \cdot \mathbf{q}}{\mathbf{n}=\frac{1.96^{2} \cdot \mathbf{1 6 3 1} \cdot \mathbf{5 0} \% \cdot \mathbf{5 0} \%}{\mathbf{d}^{2} \cdot(\mathbf{N}-\mathbf{1}) \cdot \mathbf{z}^{2} \cdot \mathbf{q}} \mathbf{2}^{2} \cdot(\mathbf{1 6 3 1 - 1 )} \cdot \mathbf{1 . 9 6} \cdot \mathbf{5 0} \% \cdot \mathbf{5 0} \%}=253
$$

El análisis estadístico de las variables cuantitativas se realizó por medio del programa SPSS V 20. 
El proceso de recolección de datos consistió en acudir a las escuelas secundarias a solicitar los permisos para realizar el estudio, una vez que se obtuvieron los permisos, se convocó a los padres de familia para firmar el consentimiento informado, al siguiente día se aplicó la encuesta con el apoyo de personal docente y administrativo de la institución, para la selección de los jóvenes que contestaron la encuesta fueron seleccionados de forma aleatoria, en cada uno de los grupos.

El análisis se realizó por medio de estadística descriptiva con medidas de tendencia central, identificar moda, media, desviación estándar, frecuencia para identificar adolescentes en las escuelas secundarias que presenten signos y síntomas de depresión.

\section{Resultados}

Aspectos sociodemográficos.

En el estudio participaron 253 adolescentes donde se observó una mayor proporción de la población en Hombres con un 59\% (Cuadro 1).

Cuadro 1. Distribución de adolescentes según género en las escuelas secundarias públicas del municipio Camerino Z Mendoza.

\begin{tabular}{lcc}
\hline Genero & Frecuencia & Porcentaje \\
\hline Masculino & 151 & $59.8 \%$ \\
Femenino & 102 & $40.2 \%$ \\
Total & 253 & $100 \%$ \\
\hline
\end{tabular}

La edad de los adolescentes está normalizada de con una media de 13.5 años y una desviación de 0.5 años véase la descripción de las frecuencias de edad en el cuadro 2.

Cuadro 2. Distribución en cifras absolutas y porcentuales de los adolescentes según la edad en escuelas secundarias públicas del municipio Camerino Z. Mendoza.

\begin{tabular}{lcc}
\hline Edad & Frecuencia & Porcentaje \\
\hline 12 años & 47 & $18.6 \%$ \\
13 años & 84 & $33.2 \%$ \\
14 años & 73 & $29.0 \%$ \\
15 años & 49 & $19.2 \%$ \\
Total & 253 & $100 \%$ \\
\hline
\end{tabular}

La proporción de adolecentes por escuela se muestra en el cuadro 3.

Cuadro 3. Distribución en cifras absolutas y porcentuales de los adolescentes pertenecientes a las escuelas secundarias públicas del municipio Camerino Z Mendoza.

\begin{tabular}{lcc}
\hline \multicolumn{1}{c}{ Escuelas } & Frecuencia & Porcentaje \\
\hline Secundaria Ignacio Manuel Altamirano & 91 & 36 \\
Escuela Secundaria Esfuerzo Obrero & 162 & 64 \\
Total & 253 & 100 \\
\hline \multicolumn{3}{c}{ Como resultado del instrumento CDI se identificó que el $100 \%$ cuenta } \\
con problemas de depresión moderado u severo, lo cual significa que existen \\
factores que afectan su desarrollo.
\end{tabular}


Cuadro 4. Niveles de depresión que presentan los adolescentes de las escuelas secundarias públicas en el municipio de Camerino Z. Mendoza.

\begin{tabular}{lcc}
\hline Variable & Frecuencia & Porcentaje \\
\hline Ausente & 0 & $0 \%$ \\
Moderado & 171 & $67.6 \%$ \\
Severo & 82 & $32.4 \%$ \\
Total & 253 & $100 \%$ \\
\hline
\end{tabular}

En el cuadro 5 se muestra que los adolescentes de género masculino que presentan un nivel moderado de depresión $60 \%$ y de género femenino $40 \%$ y en depresión severa se identifican adolescentes de género masculino $61 \%$ y en género femenino 39\%, encontrando mayor incidencia en adolescentes masculinos con un porcentaje total de $59.68 \%$ y en género femenino $40.32 \%$.

Cuadro 5. Distribución en cifras absolutas y porcentuales de género masculino y femenino con depresión moderado y severo.

\begin{tabular}{lcccccc}
\hline & \multicolumn{2}{c}{ Moderada } & \multicolumn{2}{c}{ Severa } & \multicolumn{2}{c}{ Total } \\
& $\mathrm{F}$ & $\%$ & $\mathrm{~F}$ & $\%$ & $\mathrm{~F}$ & $\%$ \\
\hline Masculino & 101 & $59.96 \%$ & 50 & $60.9 \%$ & 151 & $59.68 \%$ \\
Femenino & 70 & $40.04 \%$ & 32 & $39.1 \%$ & 102 & $40.32 \%$ \\
Total & 171 & $100 \%$ & 82 & $100 \%$ & 253 & $100 \%$ \\
\hline
\end{tabular}

Analizando los resultados del inventario de depresión infantil por medio de las edades se pudo identificar que las edades más vulnerables son de 13 y 14 años coincidiendo en los índices más altos de depresión moderado severo en e cuadro 6 se presentan los datos.

Cuadro 6. Relación entre la edad y niveles de depresión de los adolescentes de escuelas secundarias públicas del municipio Camerino Z. Mendoza.

\begin{tabular}{lcccccc}
\hline Edad & \multicolumn{7}{c}{ Moderado } & \multicolumn{2}{c}{ Inventario depresión infantil } \\
& 34 & $19.88 \%$ & 13 & $16 \%$ & Total & $\%$ \\
\hline 12 años & 60 & $35.01 \%$ & 24 & $29 \%$ & 84 & $33.2 \%$ \\
13 años & 46 & $27 \%$ & 27 & $33 \%$ & 73 & $29 \%$ \\
14 años & 31 & $18.02 \%$ & 18 & $22 \%$ & 49 & $19.3 \%$ \\
15 años & 171 & $100 \%$ & 82 & $100 \%$ & 253 & $100 \%$ \\
Total & $17 \%$
\end{tabular}

Realizando el análisis de resultados de los adolecentes que viven con personajes de la familia se observó que el mayor índice de depresión es cuando en el hogar están los padres (papá y mamá) y hermanos, seguido de adolecentes que viven con otras personas que no son los padres. Por otro lado, el menor índice identificado es cuando el padre vive con los hijos, los datos se presentan en el cuadro 7. 
Cuadro 7. Personas con quien viven los adolescentes de las escuelas públicas del municipio Camerino Z. Mendoza.

\begin{tabular}{lcccccc}
\hline Personajes de la familia & \multicolumn{2}{c}{ Moderada } & \multicolumn{2}{c}{ Severa } & F & $\%$ \\
\hline Madre & 7 & $4.1 \%$ & 4 & $4.88 \%$ & 11 & 5.5 \\
Mamá y papá & 6 & $3.5 \%$ & 2 & $2.44 \%$ & 8 & 3 \\
Mamá, papá y hermanos & 75 & $43.9 \%$ & 38 & $46.34 \%$ & 113 & 44 \\
Mamá y hermanos & 19 & $11.1 \%$ & 3 & $3.65 \%$ & 22 & 9 \\
Papá y hermanos & 3 & $1.7 \%$ & 1 & $1.22 \%$ & 4 & 1.5 \\
Papás, hermanos y abuelos & 26 & 15.2 & 11 & $13.42 \%$ & 37 & 4 \\
Otros & 35 & $20.5 \%$ & 23 & $28.05 \%$ & 58 & 22.9 \\
Total & 171 & $100 \%$ & 82 & $100 \%$ & 253 & 100 \\
\hline
\end{tabular}

Los resultados de depresión con base a las escuelas analizadas se observaron que la escuela Esfuerzo Obrero, tiene mayores índices de depresión (moderado y severo), por lo que se cree necesario como recomendación realizar un programa en la institución para tratar la depresión.

Cuadro 8. Niveles de depresión en las escuelas secundarias públicas del municipio Camerino Z Mendoza.

\begin{tabular}{lcccccc}
\hline \multirow{2}{*}{ Nombre de la escuela } & \multicolumn{5}{c}{ Inventario depresión infantil } \\
& Moderado & Severo & Total & $\%$ \\
\hline Secundaria Ignacio Manuel Altamirano & 68 & $40 \%$ & 23 & $29 \%$ & 91 & 36 \\
Escuela Secundaria Esfuerzo Obrero & 103 & $60 \%$ & 59 & $71 \%$ & 162 & 64 \\
$\quad$ Total & 171 & 100 & 82 & 100 & 253 & $100 \%$ \\
\hline
\end{tabular}

Finalmente, como parte de los resultados de los estudiantes en relación a la afinidad o compromiso que sienten por los profesores, debido a que los motivan o apoyan para seguir adelante son: los adolecentes que aseguraron no tener afinidad con ninguno tiene mayores índices de depresión. En caso de depresión moderada se identificaron que las materias de matemáticas, español e historia son las que tienen mayores porcentajes, y en el caso de depresión severa se observa que las materias con mayor porcentaje son matemáticas e ingles, véase el cuadro 9.

Cuadro 9. Resultados variables EESA

\begin{tabular}{lcccccc}
\hline \multicolumn{1}{c}{ EESA } & \multicolumn{2}{c}{ Moderado } & \multicolumn{2}{c}{ Inventario depresión infantil } & \multicolumn{2}{c}{ Total } \\
\hline Ninguno & 27 & $15.8 \%$ & 22 & $26.8 \%$ & 49 & $19.36 \%$ \\
Matemáticas & 27 & $15.8 \%$ & 9 & $11 \%$ & 36 & $14.23 \%$ \\
Español & 22 & $12.9 \%$ & 6 & $7.3 \%$ & 28 & $11.06 \%$ \\
Historia & 17 & $9.9 \%$ & 4 & $4.7 \%$ & 21 & $8.3 \%$ \\
Geografía & 15 & $8.7 \%$ & 6 & $7.3 \%$ & 21 & $8.3 \%$ \\
Asignatura Estatal & 15 & $8.7 \%$ & 4 & $4.8 \%$ & 19 & $7.5 \%$ \\
Inglés & 11 & $6.4 \%$ & 7 & $8.53 \%$ & 18 & $7.12 \%$ \\
Formación Cívica y Ética & 9 & $5.2 \%$ & 5 & $6.63 \%$ & 14 & $5.53 \%$ \\
Educación física & 7 & $4.2 \%$ & 3 & $3.65 \%$ & 10 & $3.95 \%$ \\
Taller & 5 & $2.9 \%$ & 5 & $6.09 \%$ & 10 & $3.95 \%$ \\
Ciencias & 5 & $2.9 \%$ & 1 & $1.2 \%$ & 6 & $2.37 \%$ \\
Química & 4 & $2.4 \%$ & 4 & $4.8 \%$ & 8 & $3.16 \%$
\end{tabular}




\begin{tabular}{lcccccc} 
Biología & 4 & $2.4 \%$ & 2 & $2.4 \%$ & 6 & $2.37 \%$ \\
Otro & 3 & $1.8 \%$ & 4 & $4.8 \%$ & 7 & $2.8 \%$ \\
Total & 171 & $100 \%$ & 82 & $100 \%$ & 253 & $100 \%$ \\
\hline
\end{tabular}

EESA: ¿En la escuela sientes afinidad por algún maestro o maestra porque te sientes comprometido, aceptado y/o motivado a salir adelante por él o ella?

\section{Conclusión}

Si bien existen programas de gobierno que intentan prevenir temas de salud como: la obesidad, diabetes, acoso, violencia, entre otros. No existe difusión de las cifras en gobiernos municipales, y tampoco se trabajan en estrategias para mitigar esos fenómenos sociales como es el caso de la depresión.

Debido a la escasez de evidencias existentes en el tema de depresión en adolescencia, se realizó un trabajo exploratorio y descriptivo con la finalidad de identificar que variables sociodemográficas son las que más inciden en la depresión.

Las variables que presentan mayor incidencia son adolecentes varones, edad (13 y 14 años) que viven en compañía de sus padres y hermanos, y que a su vez no sienten apoyo o motivación por ninguno de sus profesores.

Como complemento del estudio es necesario abordar las causas por las que aun cuando los adolecentes viven con sus padres y hermanos, existe un mayor nivel de depresión (comparaciones, conflictos, valores, etc.).

Los resultados del estudio permiten hacer consideraciones para el desarrollo y elaboración de una estrategia de intervención para prevenir la presencia de depresión en los adolescentes en escuelas secundarias en el municipio de Camerino Z. Mendoza.

\section{References:}

1. Berenzon, S., Lara, M. A., Robles, R., \& Medina-Mora, M. E. (2013). Depresión: estado del conocimiento y la necesidad de políticas públicas y planes de acción en México. Salud pública de México, 55 (1), 74-80.

2. Dirección General de Planeación, Planeación y Estadística Educativa. (1 de 08 de 2019). https://www.planeacion.sep.gob.mx. Obtenido de https://www.planeacion.sep.gob.mx/principalescifras/

3. Diz, J.I. (2013). Desarrollo del adolescente: aspectos físicos, psicológicos y sociales. Pediatría integral, 17(2), 88-93.

4. Ferrer, M. L. E. (2015). El afrontamiento y la generación de estrés en la depresión en la adolescencia (Doctoral disertación, Universidad de Deusto).

5. Jiménez Tapia, A., Wagner, F., Rivera Heredia, M. E., \& GonzálezForteza, C. (2015). Estudio de la depresión en estudiantes de la Ciudad 
de México y del Estado de Michoacán por medio de la versión revisada de la CES-D. Salud mental, 38(2), 103-107.

6. López Guerrero, I. D., \& Becerril Esteban, J. (2014). Tipología y comunicación familiar en pacientes de 9 a 16 años con diagnóstico de depresión, que acuden a la consulta de psiquiatría en el hgr 220 con medicina familiar del IMSS, de octubre del 2012 a febrero del 2013.

7. Morocho Guadalima, A. P. (2014). Depresión y su relación con riesgo de suicido en los adolescentes de la Unidad Educativa José Antonio Eguiguren La Salle y el Instituto Superior Tecnológico Daniel Álvarez Burneo de la ciudad de Loja (Bachelor's thesis).

8. Ortiz Amores, A. C. (2012). Aplicación de la terapia cognitiva de beck para el tratamiento de depresión en un grupo de adolescentes de 12 a 15 años del Instituto Técnico Superior Gran Colombia de la ciudad de Quito de octubre del 2011 a enero del 2012 (Bachelor's thesis, Quito/UIDE/2012).

9. Palacios, X. (2019). Adolescencia: ¿una etapa problemática del desarrollo humano? Revista Ciencias de la Salud, 17(1), 5-8.

10. Rivera-Heredia, M. E., Martínez-Servín, L. G., \& Obregón-Velasco, N. (2013). Factores asociados con la sintomatología depresiva en adolescentes michoacanos. El papel de la migración familiar y los recursos individuales, familiares y sociales. Salud mental, 36(2), 115122.

11. Romero, N. A. R., Guajardo, J. G., Ibarra, A. F., \& Castro, S. C. F. (2013). Las competencias socioemocionales como factor protector ante la sintomatología de ansiedad y depresión en adolescentes. Psicogente, 16(29), 55-64.

12. Tesouro Cid, M., Espadalé, P., Luisa, M., Bonachera Carreras, F., \& Martínez Fernández, L. (2013). Estudio sobre el desarrollo de la identidad en la adolescencia.

13. Tijerina González, Liliana Zandra, et al. (2019). "Depresión, ansiedad y estrés en estudiantes de nuevo ingreso a la educación superior." Revista Salud Pública y Nutrición 17.4: 41-47.

14. Vivar López, M. M., \& Brito Pérez, L. P. (2015). Evaluación del Autoconcepto en adolescentes (Bachelor's thesis). 